\title{
PREVALENCIA DE INFECCION A TRYPANOSOMA CRUZI EN DONADORES DE SANGRE EN EL ESTADO DE JALISCO, MEXICO.
}

\author{
Francisco Trujillo Contreras, Felipe Lozano Kasten, M. Margarita Soto \\ Gutiêrrez y Rene Hernández Gutiérrez
}

\begin{abstract}
Durante el periodo de Octubre de 1991 a Marzo de 1992, se tomaron 3419 muestras de donadores de sangre de 12 localidades rurales y de 8 hospitales urbanos a los que se les realizó un estudio serológico mediante la reacción de hemaglutinación indirecta encontrándose anticuerpos contra Trypanosoma cruzi en 44 individuos 39 masculinos y 5 femininos. El $90,9 \%$ de donantes fueron masculinos. De acuerdo a su procedencia, el 73,5\% fué del área urbana y el 26,5\% del área rural. De acuerdo a los resultados el riesgo de transmisión de $\mathrm{T}$. cruzi por transfusión sanguinea está latente por la creciente urbanización de la enfermedad de Chagas.
\end{abstract}

Palabras-claves: Bancos de sangre. Donadores infectados. Trypanosoma cruzi. Enfermedad de Chagas.

La enfermedad de Chagas es ocasionada por el Trypanosoma cruzi, protozoario hemoflagelado que en zonas endemicas generalmente se transmite al hombre por la picadura-deyección de insectos hematofagos de la familia Reduviidae. Estes arrojan trypomastigotes metaciclicos que entran en el cuerpo humano por el sitio de la picadura sufriendo transformaciones en sus fases de mastigotes y trypomastigotes según el sitio del cuerpo donde se encuentren y ocasionando de esta manera las fases aguda o crónica de la enfermedad ${ }^{3}$.

Sin embargo otro mecanismo importante de transmisión de esta enfermedad es a través de la transfusión sanguinea, ya que los individuos serológicos positivos generalmente son portadores de parásitos en su circulación sanguinea. Cerisola y cols $^{2}$ verificaron que el riesgo de infección aumenta por esta via porque el parásito puede permanecer viable hasta 12 dias en las condiciones en que habitualmente se conserva la sangre en los bancos de sangre.

Em México, Hoffman en la decada de los 20 estudia la relación de los triatomas con la

\footnotetext{
Centro de Docencia, Investigación y Diagnóstico de Enfermedades Tropicales. Universidad de Guadalajara. Jalisco, México.

Endereço para correspondência: Dr. Francisco Trujillo Contreras. Carlos Merida 348. Colinas de la Normal. 44270 Guadalajara, Jalisco, México.

Recebido para publicação em 20/01/93
}

enfermedad de Chagas $^{6}$, más tarde Mazzotti ${ }^{7}, y$ Biagi ${ }^{1}$, incrementan ese conocimiento sobre la taxonomía y distribución geográfica, iniciando estudios en casos humanos y logrando descubrir los dos primeros en 1940, la virulencia de las cepas de T. cruzi, los reservorios del parásito e inclusive los enemigos naturales de la chinche ${ }^{8}$.

En el Estado de Jalisco, Cuartero y Ponce 4 publicaron en 1967 dos casos de enfermedad de Chagas que detectaron en la campaña de erradicación de paludismo en los municipios de Chimaltitán y Santa María de los Angeles. Esta fué la primera información sobre esta enfermedad en el estado.

En 1974 Velazco Castrejon y cols publicaron 3 nuevos casos humanos de enfermedad de Chagas aguda en los municipios de Santa Ana Acatlán de Juarez y Atotonilco el Alto ${ }^{18}$. En 1976 Tellache $^{12}$ publicó una recopilación de los casos descubiertos por la Comisión Nacional de Erradicación de Paludismo en México, entre ellos 14 de Jalisco, algunos publicados previamente por otros autores ${ }^{14}$ 16. En 1979 Tay y cols ${ }^{11}$ refirieron haber encontrado 8 casos humanos nuevos mediante observación microscopica de sangre periférica de habitantes del municipio de Zacoalco de Torres, Jalisco. En el año de 1986 se descubren 11 casos agudos en el municipio de Tuxcueca, 2 en Sayula y otros 2 en el municipio de Cocula con lo que Jalisco alcanzó el primer lugar en el país ${ }^{5}{ }^{16}$. Así mismo a fin de 1986 Velazco y Trujillo Contreras estudiaron un caso de megacolon 
Contreras FT, Kasten FL, Gutiérrez MMS, Gutiérrez RH. Prevalencia de infeccion a Trypanosoma cruzi en donadores de sangre en El Estado de Jalisco, México. Revista da Sociedade Brasileira de Medicina Tropical 26:89-92, abr-jun, i993.

chagásico comprobado por xenodiagnóstico, cuya infección se originó en el rancho de San Nicolás municipio de Tuxcueca el cual sugiere la presencia de la cepa $\mathrm{Z} 2$ de $T$. cruzi en México ${ }^{16}$.

A raiz de estos antecedentes, la Universidad de Guadalajara decidió realizar en 1987 un estudio seroepidemiológico para conocer la prevalencia de la enfermedad de Chagas en los 124 municipios del estado obteniendo una tasa de 21,6 por 100 habitantes ${ }^{13}$. Los datos obtenidos por la Universidad de Guadalajara concuerdan con las pruebas serológicas reportadas para algunos municipios en una publicación hecha por Velazco y Trujillo Contreras en $1989^{16}$.

Ante estos acontecimientos se hizo una divulgación de los hechos por la prensa que generó en gran alarma en la población Jaliscience y se determinó nombrar a Jalisco como una zona endémica de Chagas, razón por la cual consideramos importante investigar el riesgo de transmisión de esta enfermedad a través de la transfusión sanguinea mediante una encuesta serológica de la infección por $T$. cruzi en los donadores de banco de sangre en el estado de Jalisco, México.

C'jjetivos: - Realizar busqueda de anticuerpos para $T$. cruzi a los donadores de sangre del estado de Jalisco; - testar prueba de hemaglutinación indirecta con antigeno donado por el Instituto Mario Fatala Chaben de Buenos Aires, Argentina.

\section{MATERIAL Y METODOS}

Durante Octuhre de 1991 a Marzo de 1992 se tomaron al azar muestras de sangre de los donantes que acudieron en los bancos de sangre de los siguientes lugares: Ameca, Autlán, Casimiro Castillo, Ciudad Guzmán, Ocotián, Tala, Tamazula, Tequila, Magdalena, Tepaíitlán, Puerto Vallarta y Yahualica (12 localidades rurales) y de los Hospitales Generales de Zona ${ }^{\circ} 14,89,45$ y 46, GinecoObstetricia, Pediatría y Centro Médico de Occidente del IMSS y el Nuevo Hospital Civil de Guadalajara ( 8 hospitales urbanos) de ellos se utilizó el suero al cual se le practico un estudio serológico mediante la reacción de hemaglutinación indirecta con antigeno donado por el Instituto Mario Fatala Chaben de Buenos Aires, Argentina.

\section{Fundamentos del método}

Los individuos que padecen enfermedad de Chagas crónica poseen en el suero anticuerpos del tipo IgG específicos contra los antígenos citoplasmáticos del $T$. cruzi. Tales anticuerpos se pueden encontrar en concentraciones variables, siendo posible su detección por técnicas inmunológicas tales como la hemaglutinación indirecta (HAI). La HAI se basa en la propiedad que tienen los anticuerpos (que en este caso son anti- $T$. cruzi) de producir aglutinación específica en presencia de globulos rojos sensibilizados con los correspondientes antigenos.

Como en el suero, tanto de indivíduos infectados como de los no infectados, existen anticuerpos inespecíficos, debe investigarse su presencia, especialmente para los anticuerpos heterófilos que son capaces de aglutinar glóbulos rojos de diferentes especies. Dicha investigación se realiza enfrentando el suero con glóbulos rojos no sensibilizados los anticuerpos interferentes se eliminan mediante tratamiento de 2-mercapto-etanol.

El procedimiento de la técnica se realizó siguiendo las indicaciones del manual de procedimientos del Instituto Mario Fatala Chaben de Argentina.

\section{RESULTADOS}

Del total de 3419 muestras, $310(9,07 \%)$ fueron mujeres y $3109(90,93 \%)$ hombres. La edad de los donantes estuvo comprendida entre los 18 y 51 años con un número mayor de individuos jovenes (45\%) - de 21 a 30 años - (Tabla 1) según la procedencia de los donantes se dividieron en urbanos y rurales (Tabla 2).

Los resultados de la HAI se exponen en la Tabla 3 , de las 3419 muestras, 44 (1,28\%) resultaron positivas. Se muestran resultados obtenidos por procedencia de donantes. El porcentaje de positividad para la zona urbana fué de 1,31 y para la rural de 1,21 (Tablas 1, 2 y 3). 
Contreras FT, Kasten FL, Gutiérrez MMS, Gutiérrez RH. Prevalencia de infeccion a Trypanosoma cruzi en donadores de sangre en El Estado de Jalisco, México. Revista da Sociedade Brasileira de Medicina Tropical 26:89-92, abr-jun, 1993.

Tabla 1 - Distribución porcentual por grupos de edad y sexo de los donantes de sangre estudiados en El Estado de Jalisco, Mexico, 1991-1992.

\begin{tabular}{|c|c|c|c|c|c|c|c|c|}
\hline \multirow{3}{*}{ Grupo de edad } & \multicolumn{4}{|c|}{ Sexo } & & & & \\
\hline & \multicolumn{2}{|c|}{ Masculino } & \multicolumn{2}{|c|}{ Femenino } & \multicolumn{2}{|c|}{ Total } & \multicolumn{2}{|c|}{ Positivos } \\
\hline & $n^{0}$ & $\%$ & $n^{0}$ & $\%$ & $n^{0}$ & $\%$ & $n^{0}$ & $\%$ \\
\hline $18-20$ & 489 & 14,30 & 60 & 1,75 & 549 & 16,10 & - & - \\
\hline $21-30$ & 1539 & 45,00 & 171 & 5,00 & 1710 & 50,00 & 28 & 0,81 \\
\hline $31-40$ & 749 & 21,90 & 73 & 2,14 & 822 & 24,00 & 8 & 0,23 \\
\hline $41-50$ & 313 & 9,15 & 5 & 0,15 & 318 & 9,30 & 7 & 0,20 \\
\hline$\geqslant 51$ & 19 & 0,56 & 1 & 0,03 & 20 & 0,60 & 1 & 0,02 \\
\hline Total & 3109 & 90,90 & 310 & 9,07 & 3419 & 100,00 & 44 & 1,28 \\
\hline
\end{tabular}

Tabla 2 - Prevalencia de serología positiva de los donadores de sangre en el Estado de Jalisco, México, según procedencia, 1991-1992.

\begin{tabular}{lrrrrr}
\hline \multirow{2}{*}{ Procedencia } & \multicolumn{2}{c}{ Total } & & \multicolumn{2}{c}{ Positivos } \\
\cline { 2 - 3 } \cline { 6 - 7 } & \multicolumn{1}{c}{$\mathrm{n}^{\mathbf{0}}$} & $\%$ & & $\mathrm{n}^{\mathrm{0}}$ & $\%$ \\
\hline Urbana & 2514 & 73,50 & & 33 & 1,31 \\
Rural & 905 & 26,50 & & 11 & 1,21 \\
\hline Total & 3419 & 100,00 & & 44 & 2,52 \\
\hline
\end{tabular}

\section{DISCUSION}

El porcentaje de infección obtenido en los bancos de sangre que fué de $1,28 \%$ pareciera resultar muy bajo, pero si se toma en cuenta que corresponde a gente aparentemente sana y en un estado de salud que le permite donar sangre resulta verdaderamente alarmante. La mayor prevalencia de infección en el área urbana se debe a que los donantes urbanos correspondieron al 73,5\% y los rurales a un $26,5 \%$ pero llama la atención que de 905 rurales 11 fueron positivos contra 33 urbanos de 2514 donantes. Sin embargo las grandes ciudades no escapan del riesgo de la infección chagásica por transfusiones debido a la progresiva migración rural de personas infectadas, fenómeno que ha llevado a una creciente urbanización de la enfermedad de Chagas en el estado.

Mediante el empleo del xenodiagnóstico, se ha demostrado que en la enfermedad de Chagas crónica alrededor del $50 \%$ de inviduos infectados
Tabla 3 - Distribución porcentual por sexo de 3419 donadores positivos a enfermedad de Chagas, estudiados en Jalisco, México, 1991-1992.

\begin{tabular}{lrrrc}
\hline Sexo & $\begin{array}{c}\text { Posi- } \\
\text { tivos }\end{array}$ & $\begin{array}{c}\text { Nega- } \\
\text { tivos }\end{array}$ & Total & $\begin{array}{c}\text { Positivos } \\
\%\end{array}$ \\
\hline $\begin{array}{l}\text { Masculino } \\
\text { Femenino }\end{array}$ & 39 & $\begin{array}{r}3070 \\
305\end{array}$ & $\begin{array}{c}3109 \\
310\end{array}$ & $\begin{array}{c}1,25 \\
1,61\end{array}$ \\
\hline Total & 44 & 3375 & 3419 & 1,28 \\
\hline
\end{tabular}

presentaron tripomastigotes de $T$. cruzi circulantes ${ }^{10}$.

La política de control de la infección en los bancos de sangre se podría enfocar bajo dos aspectos, de acuerdo con las recomendaciones oficiales ${ }^{19}$ : a. determinar mediante serología a aquellos donantes infectados para descartarlos y b. discriminar la sangre obtenida y tratarla cuando resulte seropositiva con sustancias tripanomicidas (violeta de genciana diluida 1:4000).

\section{SUMMARY}

A Chagas Disease serological study was done from October 1991 to March 1992 and 3419 samples were taken from people who donated blood at 12 county areas of Jalisco, México and 8 urban hospitals, by means of indirect hemagglutination reaction. The results indicate that: $73.5 \%$ of the donors were from urban area, $26.5 \%$ were from rural areas; $1.28 \%$ of the donors $(N=44)$ were considered infected. Thirty nine of them (1.14) were males and 5 females. According to the above mentioned data, we can confirm that the risk of transmission of 
Contreras FT, Kasten FL, Gutiérrez MMS, Gutiérrez RH. Prevalencia de infeccion a Trypanosoma cruzi en donadores de sangre en El Estado de Jalisco, México. Revista da Sociedade Brasileira de Medicina Tropical 26:89-92, abr-jun, 1993.

Trypanosoma cruzi can occur by blood transfusion and this is potentially latent because of the growing urbanization of Chagas disease.

Key-words: Blood banks. Infected donors. Trypanosoma cruzi. Chagas disease.

\section{REFERENCIAS BIBLIOGRÁFICAS}

1. Biagi F. Foco epidémico de enfermedad de Chagas en México. Tetitlán, Guerrero. Revista Facuitad Medicna 6:625-631, 1964.

2. Cerisola JA, Ravinoch A, Alvarez M, Li Coretto $\mathrm{CH}$, Pruneda J. Enfermedad de Chagas y la transfusión sanguinea. Boletín de la Oficina Sanitaria Panamericana 73:203-221, 1972.

3. Chagas $C$. Nova tripanozomiaze humana. Imprensa Médica 17:154-155, 1909.

4. Cuartero LM, Ponce D, Recco R. Cinco nuevos casos de enfermedad de Chagas en Zacatecas y Jalisco, en la República Mexicana. Revista de Investigación de Salud Pública 27:29-36, 1967.

5. Hernandez MI, Ericson $\mathrm{CH}$, Delgadillo $C$. New focus of Chagas disease in México. Lancet 100:23, 1987.

6. Hoffman C. Nota acerca de un probable transmisor de tripanosomiasis humana en el estado de Veracruz.

Revista Mexicana Biología B:12-18, 1928.

7. Mazzoti L. Investigación sobre la existencia de la enfermedad de Chagas en el país. Demostración de tripanosomas de Reduvidos transmisores. Revista Mexicana de Medicina 16:584-585, 1936.

8. Mazzoti L. Infección natural de Trypanosoma cruzi en Chagas, en Triatoma dimidiata (Ctreille) de los estados de Yucatán, Campeche, Chiapas, Veracruz y Jalisco. Revista Mexicana de Medicina 17:283286, 1937.

9. Mazzoti L. Dos casos de enfermedad de Chagas en el estado de Oaxaca, México. Gaceta Médica Mexicana 70:417-420, 1940.

10. Schenone $H$, Alfaro E, Reyes H, Taucher E. Valor del xenodiagnóstico en la infección chagásica crónica.
Boletín Chileno de Parasitología 23:149-154, 1958.

11. Tay J, Salazar Shetino PM, Haro I, De Garcia Y, Gutiérrez M. Estudio epidemiológico de la enfermedad de Chagas en el estado de Jalisco, México. Revista de Salud Pública de México 2:145$149,1979$.

12. Tellaeche AM. Hallazgo de Trypanosoma (Schizotrypanum) en muestras de sangre tomadas a febriles del área palúdica de México. Dirección General de Investigación en Salud Pública. Boletín Informativo $7 / 8: 1-5,1979$.

13. Trujillo Contreras F, Martinez Orozco LC, Hernández R, Lozano F, Ruvalcaba S. Estudio epidemiológico de la prevalencia de la enfermedad de Chagas en el estado de Jalisco, México - 1987 (Reporte preliminar). Revista de la Asociación Medica de Jalisco, Nueva Epoca 3:28-31, 1988.

14. Velazco Castrejon O, Garcia LA. Estudio clinico y epidemiológico de un nuevo caso humano de enfermedad de Chagas en Jalisco, México. Prensa Médica Mexicana 35:438-440, 1970.

15. Velazco $O$, Guzman C. Importancia de la enfermedad de Chagas en México. Revista Latinoamericana de Microbiología 28:275-283, 1986.

16. Velazco O, Ramirez J, Sanchez B, Trujillo F, Guzman C. La enfermedad de Chagas en Jalisco, México. Revista Mexicana de Parasitología 2:2932, 1989.

17. Velazco O, Romero RL, Mendiola JG. Contribución al estudio de la enfermedad de Chagas en México. Observaciones epidemiológicas en Tepechitlán, Zacatecas. Revista de la Investigación en Salud Pública, México 30:197-204, 1970.

18. Velazco O, Tay S, Luna A. La enfermedad de Chagas en el estado de Jalisco, República Mexicana. Presentación de tres nuevos casos humanos. Revista de la Investigación en Salud Pública, México 34:107$113,1974$.

19. World Health Organization. Control of Chagas disease. Report of a WHO Expert Committee. Geneva. World Health Organization. Technical Report Series 811, 1991. 